\title{
Wire tomography in the H-1NF heliac for investigation of fine structure of magnetic islands
}

\author{
Santhosh T. A. Kumar, ${ }^{\text {a) }}$ Boyd D. Blackwell, and Jeffrey H. Harris ${ }^{\text {b) }}$ \\ Plasma Research Laboratory, Research School of Physical Sciences and Engineering, \\ The Australian National University, Canberra ACT 0200, Australia
}

(Received 19 September 2006; accepted 28 November 2006; published online 4 January 2007)

\begin{abstract}
Electron beam wire tomography in the H-1NF heliac enables high resolution mapping of vacuum flux surfaces with minimal disruption of the plasma operations schedule. Recent experimental results have proven this technique to be a highly accurate and high resolution method for mapping vacuum magnetic islands. Islands of width as small as $\delta \sim 8 \mathrm{~mm}$ have been measured, providing estimates of the internal rotational transform of the island. Point-to-point comparison of the mapping results with computer tracing, in conjunction with an image warping technique, enables systematic exploration of magnetic islands and surfaces of interest. Recent development of a fast mapping technique significantly reduced the mapping time and made this technique suitable for mapping at higher magnetic fields. This article presents recent experimental results and associated techniques. (C) 2007 American Institute of Physics. [DOI: 10.1063/1.2424453]
\end{abstract}

\section{INTRODUCTION}

Rational magnetic surfaces in stellarators can develop into magnetic islands. ${ }^{1}$ Formation of these islands can significantly modify the plasma confinement properties of the device. Characterization of vacuum magnetic islands provides information about errors of magnetic field structure, essential in verifying the magnetic geometry of a newly constructed device. Accurate mapping of vacuum magnetic flux surfaces and islands is crucial for an in-depth understanding of these issues. Many different techniques have been used to map flux surfaces on different machines worldwide. These include emissive filament technique, fast rotating fluorescent wire, and resistive wire systems in the Auburn torsatron, ${ }^{2,3}$ a fluorescent mesh technique on the compact helical system, ${ }^{4}$ a phosphor-coated screen method on the ATF torsatron, ${ }^{5}$ a fluorescent rod technique on the W7-AS stellarator, ${ }^{6}$ an image-intensifying fluorescent probe on SHEILA, ${ }^{7}$ and a movable fluorescent rod on the $\mathrm{H}-1 \mathrm{NF}$ heliac. ${ }^{8,9}$

Electron beam multiwire tomography in the H-1NF heliac ${ }^{10-12}$ provides high resolution imaging of flux surfaces. Unlike many other techniques, this is free from inaccuracies arising from large electron drifts and optical distortion arising from viewing optics and nonideal viewing angles. As low electron energies are used $(\sim 20 \mathrm{eV}$, compared to $100-350 \mathrm{eV}$ in other techniques), drift effects are minimized (important at lower magnetic fields), and the mapped drift surface is a very good approximation to the actual flux surfaces. Tomographic inversion produces electron beam images directly in machine coordinates. Therefore, a point-topoint matching with computer tracing is possible. This can help in error field estimation and magnetic modeling of the device. Because individual transits are resolved in sequence,

\footnotetext{
${ }^{a}$ Electronic mail: santhosh.kumar@anu.edu.au

${ }^{b}$ Present address: Oak Ridge National Laboratory, Oak Ridge, Tennessee.
}

the rotational transform of a surface can be calculated accurately from the mapped surface. This technique has recently been specifically modified and improved for accurate mapping of magnetic islands in H-1. For example, point-to-point comparison of the mapping results with computer tracing, in conjunction with an image warping technique, enables systematic exploration of magnetic islands and surfaces of interest. Recent development of a fast mapping technique significantly reduced the time of island exploration and made this technique suitable for mapping at higher magnetic fields. Experimental techniques and recent results are presented in this artile.

Section II gives details of the experimental setup. Section III details the mapping and reconstruction. Section IV describes the image warping technique used. Section V presents the fast mapping technique. Section VI discusses critical issues of mapping islands, and Sec. VII presents our conclusions.

\section{THE DEVICE}

$\mathrm{H}-1 \mathrm{NF}$ is a medium sized heliac ${ }^{13}$ with a major radius $\sim 1 \mathrm{~m}$ and an average plasma minor radius $\sim 0.2 \mathrm{~m}$. The coil system of $\mathrm{H}-1 \mathrm{NF}$ allows the configuration to be varied across a broad range, $0.6<t<2.0$, where $t=n / \mathrm{m}$ is the rotational transform, and $n$ and $m$ are the toroidal and poloidal winding numbers, respectively. This allows accommodating or avoiding major rational surfaces and islands in the main confinement volume. ${ }^{14}$ The variation is achieved by selectively varying currents in some of the coils with respect to the central ring conductor. The two main configuration control parameters of H-1NF are (a) $\kappa_{h}=I_{\text {hw }} / I_{\text {ring }}$, ratio of the currents in helical winding to that of ring conductor, and (b) $\kappa_{v}=I_{\text {ovf }} / I_{\text {ring }}$, ratio of the currents in outer vertical field coils to that of ring conductor. Figure 1 shows the coil structure of $\mathrm{H}-1 \mathrm{NF}$ and shown in Fig. 2 are the rotational transform profiles and magnetic flux surfaces for three different magnetic 


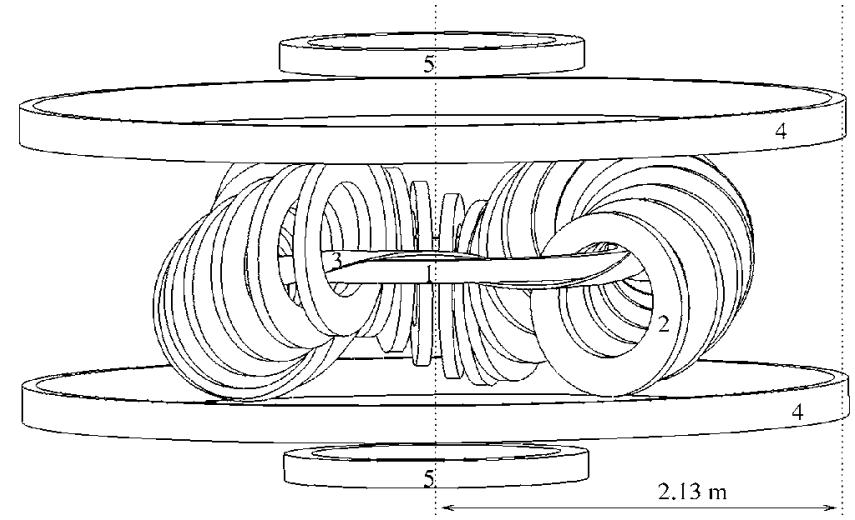

FIG. 1. Coil structure of H-1NF showing the ring conductor (1), the toroidal field coils (2), the helical winding (3), the outer vertical field coils (4), and the inner vertical field coils (5).

configurations, computed using the HELIAC code..$^{15,16}$

A typical full surface mapping using wire tomography takes 5-8 min. Thermal stresses in the coils limit the steadystate operation to lower magnetic fields $(<0.1 \mathrm{~T})$. Therefore, for most of the mapping results reported in this article, the main coil (ring conductor) current was chosen to be $\sim 1000 \mathrm{~A}(=0.07 \mathrm{~T})$. The fast mapping system enables mapping at higher coil currents (up to $6500 \mathrm{~A}=0.5 \mathrm{~T}$ ) and fast iteration to optimize the launch position for island investigations, which is discussed in Sec. V.

The electron beam for wire tomography is generated from a specially made electron gun. (Fig. 3). This uses a heated thoriated tungsten filament of length $\sim 2 \mathrm{~cm}$ and diameter $\sim 0.1 \mathrm{~mm}$. The interchangeable biasing shield has a beam aperture of $1 \mathrm{~mm}$ in diameter and a conical shaped tip which improves scanning of islands in two ways: (a) the shield diameter at the aperture is much reduced, which reduces the chance of the electron beam hitting the back of the gun in the case of near-rational surfaces and islands and (b) the projection beyond the aperture (tip) determines the smallest width of the island which can be scanned without inter-

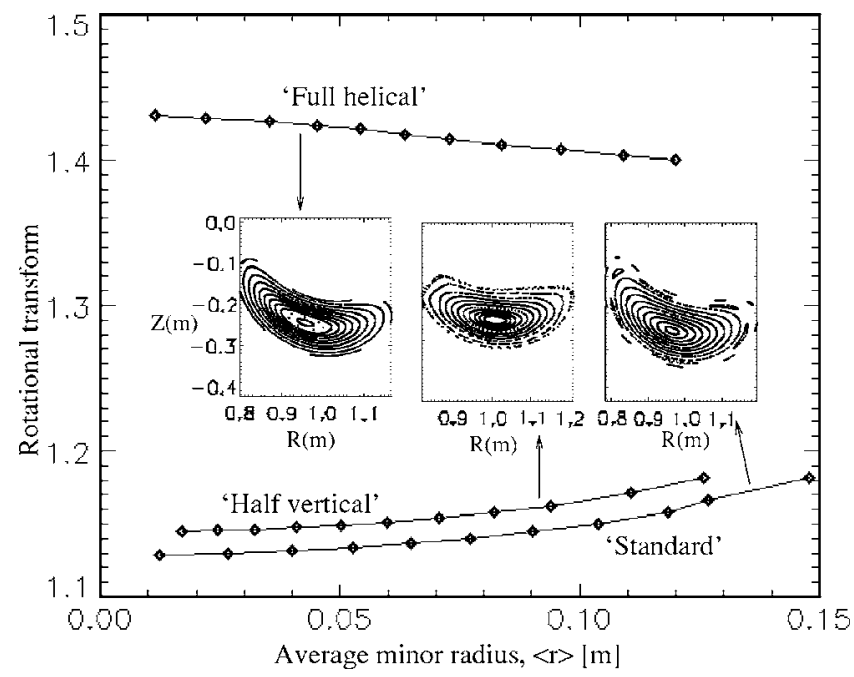

FIG. 2. Rotational transform profiles and computed flux surfaces (at $\phi$ $=85^{\circ}$ ) for three different magnetic configurations of H-1, the "full helical" $\left(\kappa_{h}=\kappa_{v}=1.0\right)$, "half vertical" $\left(\kappa_{h}=0.0, \kappa_{v}=0.5\right)$, and the "standard" $\left(\kappa_{h}\right.$ $\left.=0.0, \kappa_{v}=1.0\right)$ configurations.

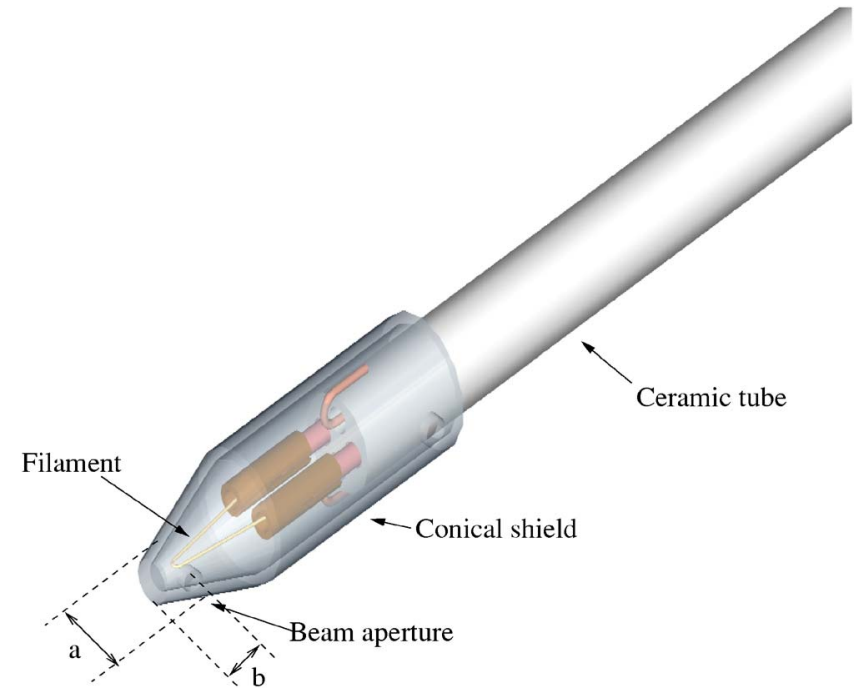

FIG. 3. (Color online) Electron gun. The shield diameter at the aperture, $a=2.9 \mathrm{~mm}$. The projection beyond the aperture (tip), $b=1.5 \mathrm{~mm}$.

secting the other side of the island. A beam current of $\sim 6-10 \mu \mathrm{A}$ is obtained with a filament current of $1.25 \mathrm{~A}$. During mapping, the base pressure of the chamber is kept as low as possible, $\leqslant 3 \times 10^{-5} \mathrm{~Pa}$, to increase the number of toroidal beam transits. Typical number of toroidal transits at this base pressure is 20-25 (this is probably also limited by the transparency of the wire grid) and the collected wire current for the first electron transit is $\sim 1 \mu \mathrm{A}$.

The multiwire assembly (described in detail in Refs. 10 and 12) consists of 64 fine molybdenum wires stretched on a circular rotating frame (Fig. 4). This is permanently installed in the $\mathrm{H}-1 \mathrm{NF}$ vacuum chamber at toroidal cross section $\phi$ $=85^{\circ}$. Rotation of this assembly with minimum wire vibrations and required angle steps is achieved by a computercontrolled microstepping stepper motor. For a typical high resolution full surface scan, the wire grid is rotated at an angle step of $\sim 0.5^{\circ}$, with a total angular rotation of $\sim 220^{\circ}$. When not in use, the whole wire assembly can be moved to "park" position, leaving the magnetic flux volume free for the plasma experiments.

\section{MAPPING AND RECONSTRUCTION}

Electrons are injected along a field line of a magnetic flux surface from the electron gun situated at toroidal angle

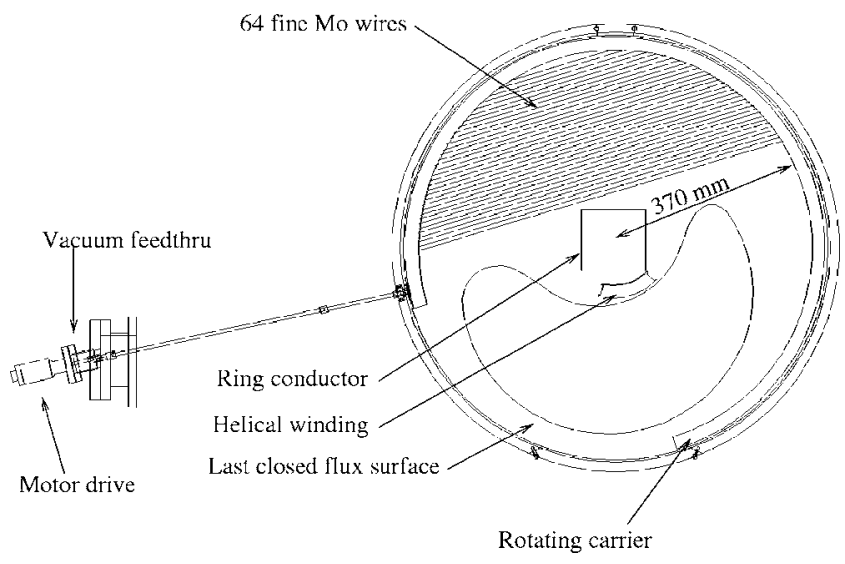

FIG. 4. Rotating multiwire assembly in park position. 

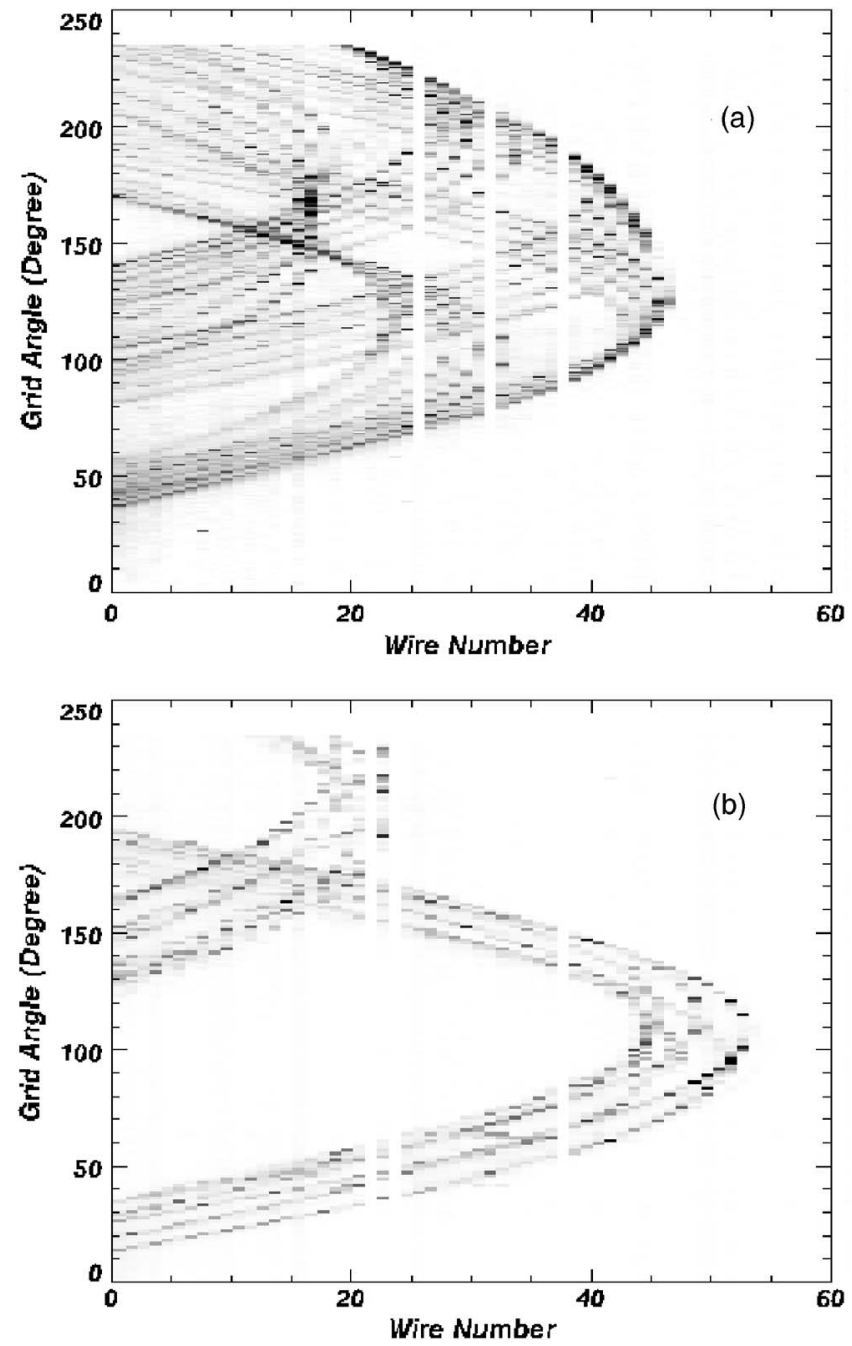

FIG. 5. Sinograms of flux surfaces for (a) "full helical" configuration $\left(\kappa_{h}\right.$ $\left.=\kappa_{v}=1.0\right)$ and (b) configuration exhibiting $m=2$ islands $\left(\kappa_{h}=\kappa_{v}=1.27\right)$.

$\phi=35.2^{\circ}$. The wire grid, which is at $\phi=85^{\circ}$, is rotated in steps (inwards or outwards) to intercept the beam transits. Current collected by each wire is recorded at each rotation angle using a 64 channel multiplexer. The data are then subjected to various inversion techniques as explained below.

In the first step of reconstruction, a sinogram is formed (collected wire current as a function of wire rotation angle and wire number). Sinograms can give fairly good information about the flux surface mapped, viz., the number of toroidal beam transits, signature of magnetic islands/rational surfaces, etc. Sinograms from two different configurations are shown in Figs. 5(a) and 5(b). Each trace (partial sinusoid) in a sinogram represents one toroidal beam transit or "puncture." It can be observed that Fig. 5(a) has five distinct groups of traces in it, indicating that this surface is near a rational surface with poloidal mode number $m$ of 5. [For a surface with $m=5$ precisely, each group reduces to one curve (transit) and the sixth transit will coincide with the first (launch) point, thus hitting the gun]. Figure 5(b) represents two $m=2$ islands with $\sim 12$ toroidal transits (which, for the three period $\mathrm{H}-1$, are connected toroidally). The clarity of this sinogram and the absence of a "cloud" effect indicate that the beam is most likely terminated by hitting the gun.
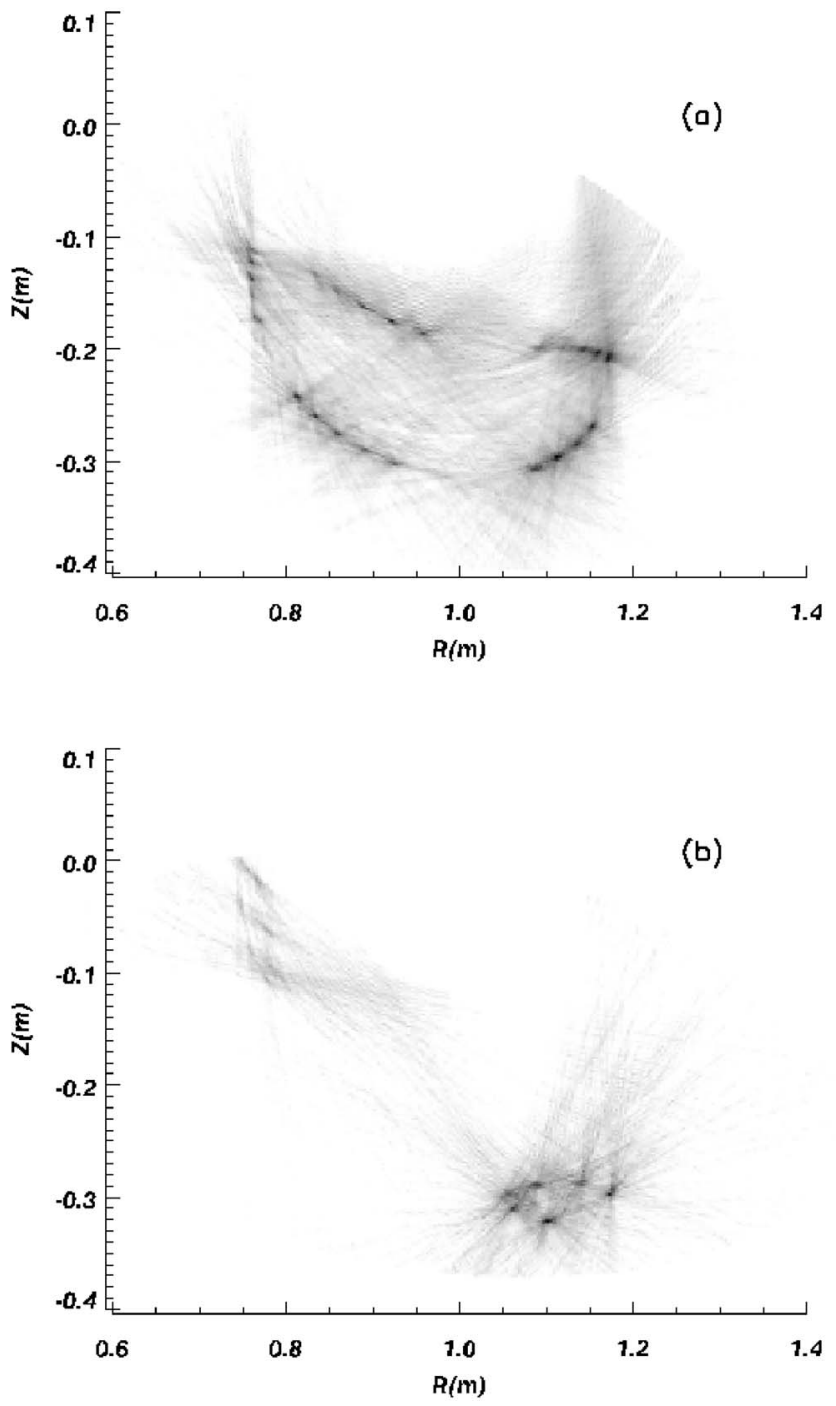

FIG. 6. Simple back projections [(a) and (b)] of data shown in Fig. 5.

The island encountered between $130^{\circ}$ and $240^{\circ}$ in Fig. 5(b) is only partially scanned, so its reconstruction in Figs. 6(b) and 7 (b) is faint and diffuse.

Two inverting techniques have been used to reconstruct the flux surface from the sinogram. Simple back projection is used for a quick analysis for an initial assessment of the puncture plot. This is a summation method, which involves distribution of the projections over an array of two dimensional pixels. The inverted image is blurred, because the projection is uniformly distributed over the pixels along the full length of each wire in the reconstruction plane [see Figs. 6(a) and 6(b)]. In order for the mapped results to be compared with field line tracing, background "noise" and the Radon transform artifacts have to be removed from the image, and each puncture point has to be separately identified. (This can be done to an extent by clipping the image.) The algebraic reconstruction technique ${ }^{17,18}$ (ART) is used for this purpose. ART is an iterative back-projection algorithm with corrections applied in each iteration to remove unwanted pixels and enhance the image. ART results for the above mentioned mappings are given in Figs. 7(a) and 7(b). Three characteristics of these data, the sparse radial grid (64 elements), its 

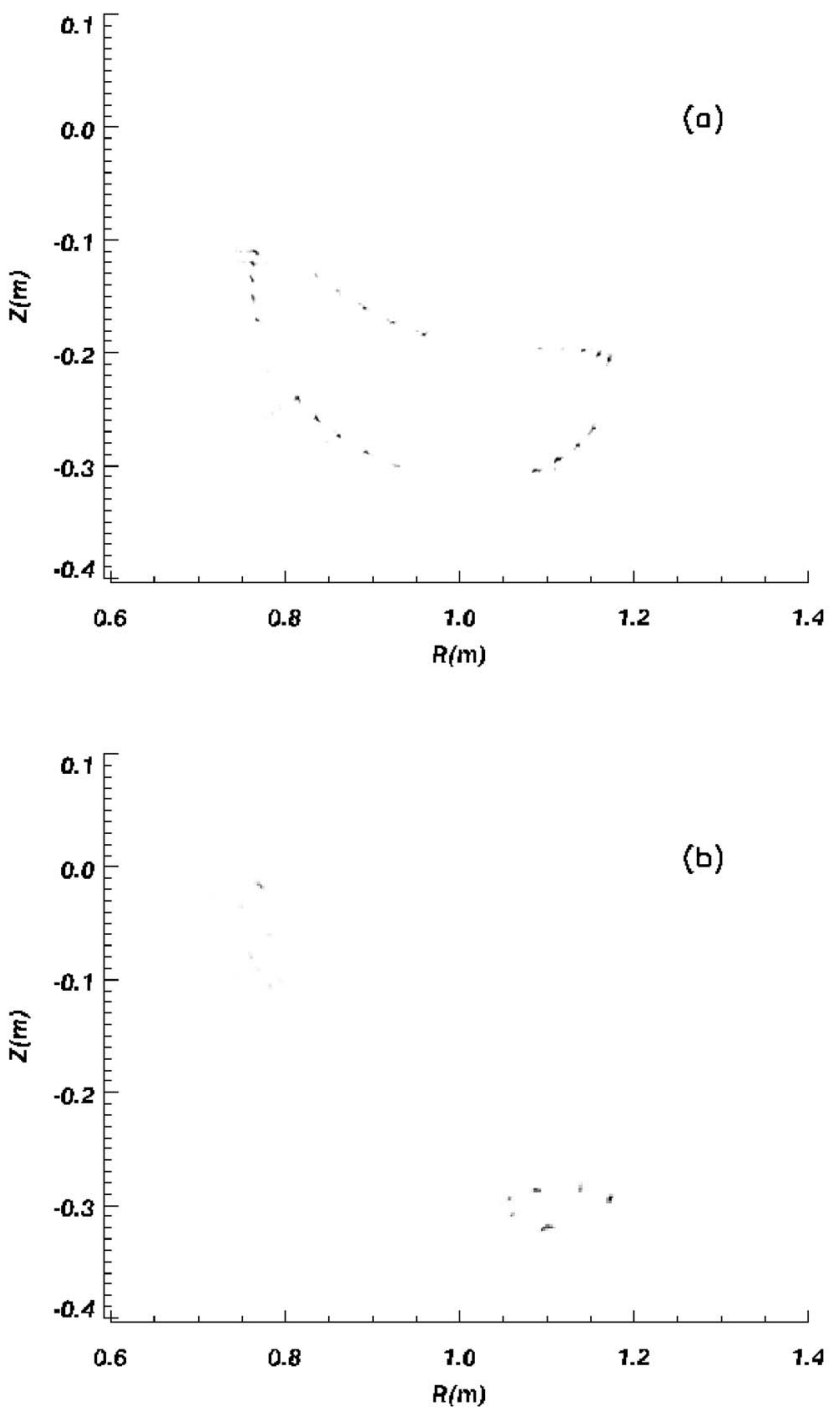

FIG. 7. ART images $[(a)$ and (b) $]$ of data shown in Fig. 5.

multiple delta function character, and the slightly perturbing nature of the measurements, prevent significant improvements by typical convolution methods used on CT x-ray data, for example, Ram-Lak, Shepp-Logan, etc. ${ }^{11,19}$

\section{IMAGE WARPING TECHNIQUE}

Being a fully three dimensional configuration, H-1NF flux surfaces have different shapes and sizes at different toroidal cross sections in any of the three periods for a particular configuration. As mentioned before, the electron gun is positioned at machine toroidal cross section $\phi=35.2^{\circ}$ and the multiwire assembly at $\phi=85^{\circ}$. Computed flux surfaces for the configuration exhibiting $m=2$ islands at the gun and wire grid locations are shown in Fig. 8. Exploring a particular flux surface, islands or island axis requires knowledge of the gun position with respect to the surface at the gun port location $\left(\phi=35.2^{\circ}\right)$. A technique has therefore been developed to warp the scanned image from $\phi=85^{\circ}$ to $\phi=35.2^{\circ}$. This uses the computed mapping of a two dimensional grid of points from one cross section to the next as a base and transforms the experimentally mapped image from one grid to the other using the "warp_tri" procedure in IDL (Research Systems,
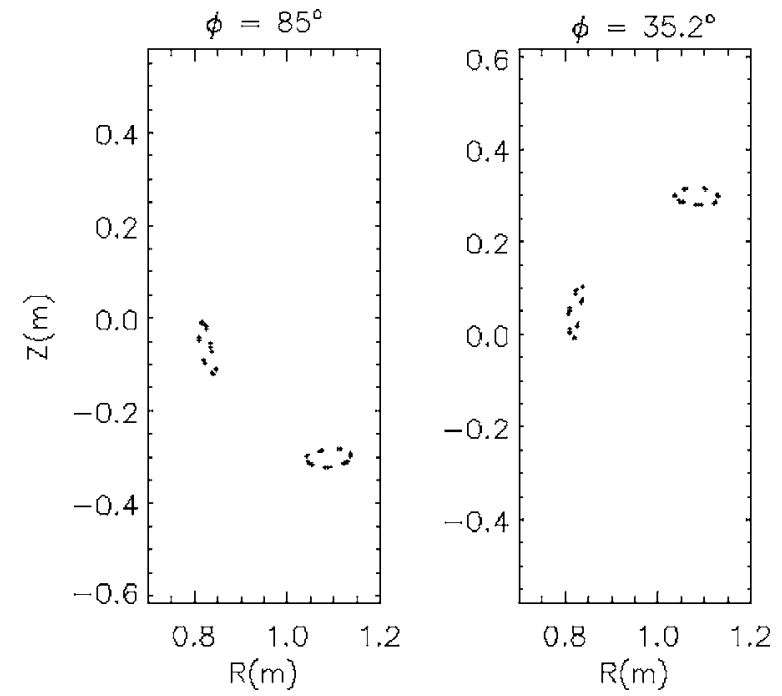

FIG. 8. Computed flux surfaces of the $m=2$ islands at the wire $\left(\phi=85^{\circ}\right)$ and the gun $\left(\phi=35.2^{\circ}\right)$ cross sections. Note that warping is not just a rotationthe thinner island at $85^{\circ}$ becomes the thicker island at $35.2^{\circ}$.

Boulder, $\mathrm{CO}$ ), which warps images based on tie points according to a Delaunay triangulation, with thin plate spline (TPS) interpolation. This technique does not rely on the existence of closed flux surfaces. Also, a highly accurate computer model is not essential as the distance over which the image is transformed is small $\left(50^{\circ}\right)$. Warped images for the "full helical" and the configuration exhibiting $m=2$ islands $\left(\kappa_{h}=\kappa_{v}=1.27\right)$, produced by a simplified threefold symmetric magnetic coil model that ignores most of the error terms and by the best fit magnetic model which includes various error field corrections, are shown in Fig. 9. Even though the magnetic flux surfaces of the simple model differ significantly from those of the best fit model (for example, the simple model for $\kappa_{h}=\kappa_{v}=1.27$ does not exhibit $m=2$ islands as the rotational transform is a little lower), the images transformed by "warping" over this short distance are accurate enough to guide the electron gun for island/surface exploration.

Shown in Fig. 10 is a warped image of the data presented in Fig. 7(b). Smearing of the warped points is due to the effect of magnetic shear on the unphysical width (in the minor radial direction) of the ART processed puncture points at the wire grid cross section. Fading of the points usually indicates beam attenuation over successive toroidal transits. Thus the first point (brightest) can be identified as the gun location. Knowledge of the gun location in relation to the local magnetic surfaces is essential to the successful optimization of the launch position, especially when mapping complicated surfaces, such as magnetic islands.

\section{FAST MAPPING}

As mentioned before, a normal full surface mapping with controlled acceleration and deceleration, and pausing the wheel for each measurement, takes 5-8 min. This makes the island exploration too time consuming and limited to low magnetic fields. (Even though the warping technique helps in guiding the electron gun movements for exploring the is- 

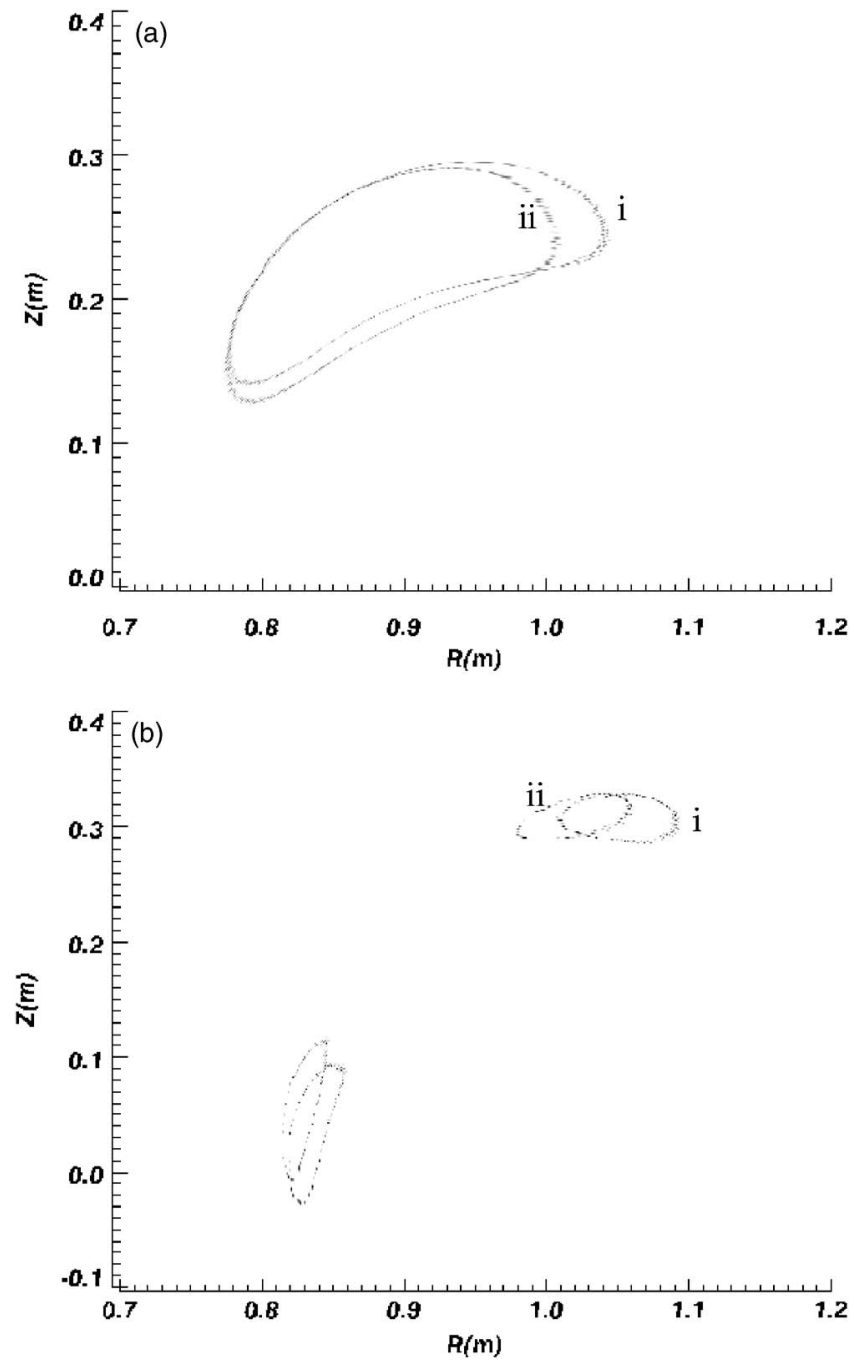

FIG. 9. Warping by the best fit (i) and the simple (ii) models. (a) "Full helical" configuration. (b) Configuration exhibiting $m=2$ islands. In practice, we use a much more accurate model than the "simple" model, and images (i) and (ii) would be almost indistinguishable from each other.

lands, it takes several full surface mappings before the optimum launch point for the island is found.) A fast mapping technique has therefore been developed. This involves continuous running of the wheel at the maximum possible speed and taking data on the run. The position (angle), time, wire number, and the wire current are recorded continuously as the wheel rotates. A high speed programmable power supply is operated in an approximately constant current mode to compensate for the induced back emf of the motor windings at high speeds. In order to make the rotation of the wheel uninterrupted during the data collection, a separate computer buffer module (combination of hardware and software) is used in controlling the stepper motor. A LABVIEW routine has been developed to perform this task.

The fast mapping technique significantly reduces the mapping time from 5-8 min to less than $15 \mathrm{~s}$ for a full surface. This is therefore very helpful in exploring the islands and also in mapping surfaces and islands at higher magnetic fields where the operation is limited to only a few seconds. Even though this method partly sacrifices the resolution of the measurement and sensitivity to fainter punctures, the re- sults indicate this technique to be almost as accurate as the normal (incremental) mapping one, especially for large surfaces. Simple back projections of surfaces with normal and fast mapping are shown in Figs. 11(a) and 11(b), respectively, for comparison. The ART processed images are much sharper, but the differences are not so apparent.

\section{CRITICAL ISSUES AND FUTURE WORK}

As mentioned above, the electron gun should be as small as possible to map islands. This requirement relates not only to the size of the islands but also to the internal rotational transform $\left(t_{i}\right)$ of the islands; the rate at which field lines twist about the island axis or $O$ point. Typical values in $\mathrm{H}-1$ range from $t_{i}=0.01$ to 0.1 . At present, the electron gun shield radius at the beam aperture is $\sim 1.5 \mathrm{~mm}$. If the distance between two adjacent beam transits (which depends on the internal rotational transform of the island) is less than or equal to $1.5 \mathrm{~mm}$, the electron beam will hit the back of the gun after just one transit. Also, if the internal rotational transform of the island is small (or, in principle, near an integer; but this has not been observed in $\mathrm{H}-1$ ), it takes many toroidal rotations for the beam to reach the other side of the island (half connection length), so that it can be unambiguously identified as an island from the mapping results. For example, it is relatively easy to detect the $m=2$ islands as the half connection length is $\sim 5-10$ transits $\left(t_{i} \sim 0.05-0.1\right)$. However, for the $m=6$ islands in $\mathrm{H} 1$, the half connection length corresponds to $\sim 65$ toroidal transits $\left(t_{i} \sim 0.006\right)$. The maximum toroidal transits we have detected so far is $\sim 25-30$. Our attempts to map $m=6$ islands have therefore been unsuccessful, even though their existence is predicted by the computer code, and we have no reason to doubt their existence.

A unique feature of the wire tomography is the point-topoint matching of the "puncture points" with the computer model. Figure 12 shows the puncture points of the mapping overlayed on the computer code results. The computer model includes current crossovers for the ring conductor (poloidal field coils), laboratory error fields, etc. A detailed error

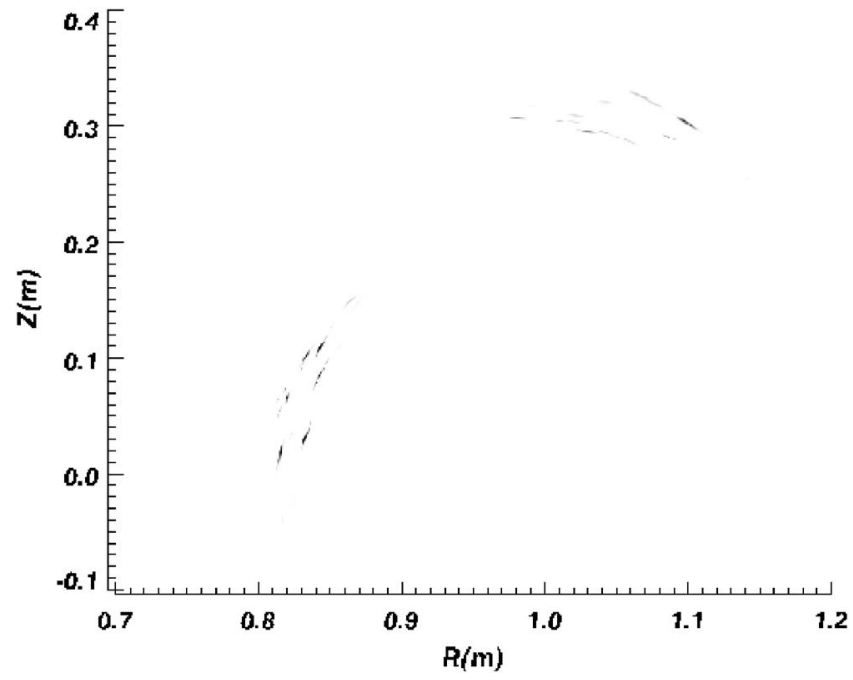

FIG. 10. Warped image of the $m=2$ islands. The original data at $\phi=85^{\circ}$ are shown in Fig. 7(b). 

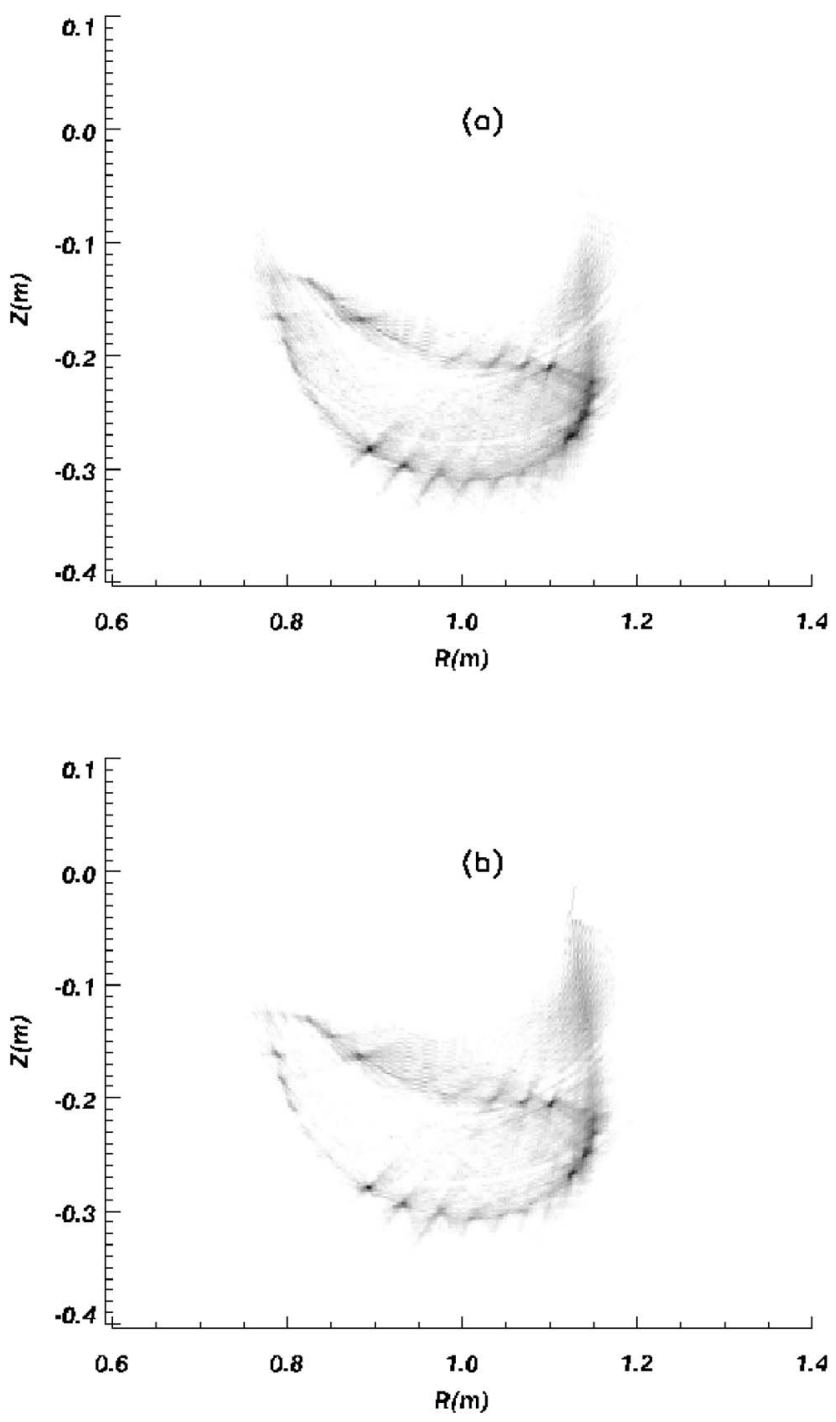

FIG. 11. Simple back projections of slow (a) and fast (b) mappings of a flux surface. The image is clipped to make the points more distinct.

analysis and computer modeling of the $\mathrm{H}-1$ magnetic configurations are being done, which will form a part of a separate paper.

\section{DISCUSSION}

Wire tomography in the H-1NF heliac offers an excellent opportunity to explore and map vacuum magnetic flux surfaces and islands with extreme accuracy. This technique has recently been specifically modified for the detection of the presence or absence of magnetic islands. An image warping technique has been developed for easy exploration of islands and their local magnetic axis. A fast mapping technique significantly reduced the time of island exploration and made mapping at higher magnetic fields possible. Islands as small as $8 \mathrm{~mm}$ have been mapped in the $\mathrm{H}-1 \mathrm{NF}$ heliac using this technique. Some interesting physics phenomena have been observed when plasma is formed in a configuration containing islands. Future work involves detailed mapping of different magnetic configurations, estimation of error fields present, and developing a best-fit magnetic model for $\mathrm{H}-1$ magnetic configurations.
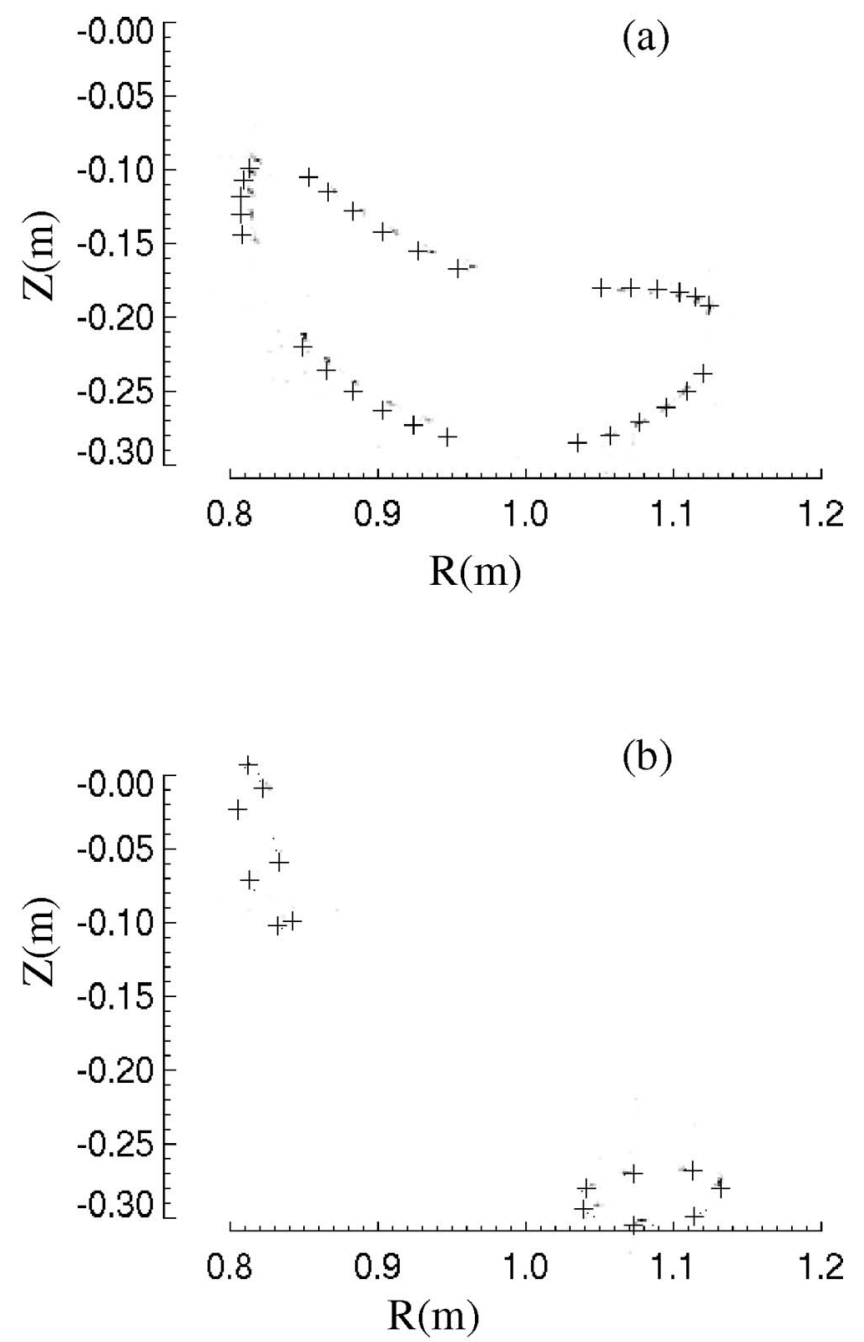

FIG. 12. Point-to-point matching of the mapped points and the computer code results ("+" points). Full helical (a) and $m=2$ islands (b).

\section{ACKNOWLEDGMENTS}

The authors would like to thank John Wach for his technical help in fabricating the electron gun and for $\mathrm{H}-1$ operation. They also thank Mark Gwynneth for developing the motor drive and LABVIEW control and data acquisition code for the standard and fast mapping system. This work was performed on the H-1NF National Plasma Fusion Research Facility established by the Australian Government, and operated by the Australian National University, with support from the Australian Research Council Grant No. DP0344361.

${ }^{1}$ A. H. Boozer, Rev. Mod. Phys. 76, 1071 (2004).

${ }^{2}$ R. F. Gandy, M. A. Henderson, J. D. Hanson, G. J. Hartwell, and D. G. Swanson, Rev. Sci. Instrum. 58, 509 (1986).

${ }^{3}$ H. Lin et al., Rev. Sci. Instrum. 66, 464 (1995).

${ }^{4}$ H. Yamada, K. Matsuoka, S. Okamura, K. Ida, and K. Nishimura, Rev. Sci. Instrum. 61, 686 (1990).

${ }^{5}$ R. J. Colchin et al., Rev. Sci. Instrum. 60, 2680 (1989).

${ }^{6}$ R. Jaenicke, E. Ascasibar, P. Grigull, I. Lakicevic, A. Weller, and M. Zippe, Nucl. Fusion 33, 687 (1993).

${ }^{7}$ T. Y. Tou, B. D. Blackwell, and L. E. Sharp, Rev. Sci. Instrum. 62, 1149 (1991).

${ }^{8}$ M. G. Shats, D. L. Rudakov, B. D. Blackwell, L. E. Sharp, and O. I. Fedyanin, Rev. Sci. Instrum. 66, 1163 (1995).

${ }^{9}$ M. G. Shats, D. L. Rudakov, B. D. Blackwell, L. E. Sharp, R. Tumlos, S. M. Hamberger, and O. I. Fedyanin, Nucl. Fusion 34, 1653 (1994). 
${ }^{10}$ R. B. Tumlos, Ph.D. thesis, The Australian National University, 1994.

${ }^{11}$ B. D. Blackwell, J. Howard, and R. B. Tumlos, Rev. Sci. Instrum. 63, 4725 (1992).

${ }^{12}$ B. D. Blackwell, R. B. Tumlos, and J. Howard, Rev. Sci. Instrum. 68, 388 (1997).

${ }^{13}$ S. M. Hamberger, B. D. Blackwell, L. E. Sharp, and D. B. Shenton, Fusion Technol. 17, 123 (1990).

${ }^{14}$ J. H. Harris, L. Cantrell, T. C. Hender, B. A. Carreras, and R. N. Morris, Nucl. Fusion 25, 623 (1985).
${ }^{15}$ A. B. Ehrhardt, The HELIAC Users Guide (Princeton Plasma Physics Laboratory, Princeton, NJ, 1985).

${ }^{16}$ B. D. Blackwell, B. F. McMillan, A. C. Searle, and H. J. Gardner, Comput. Phys. Commun. 142, 243 (2001).

${ }^{17}$ A. C. Kak and M. Slaney, Principles of Computerized Tomographic Imaging (IEEE Press, New York, 1988).

${ }^{18}$ R. Gordon, IEEE Trans. Nucl. Sci. NS-21, 78 (1974).

${ }^{19}$ J. Howard, J. Opt. Soc. Am. A 5, 999 (1988). 